\title{
THE IMPACT OF STATIN PRETREATMENT ON ACUTE DWI INFARCT PATTERN IN SYMPTOMATIC INTERNAL CAROTID ARTERY DISEASE
}

Valentin Held ${ }^{1}$, Pauline Harter ${ }^{1}$, Alex Förster ${ }^{2}$, Achim Gass ${ }^{1}$, Michael Platten ${ }^{1}$, Karin Janetzko ${ }^{3}$, Kristina Szabo ${ }^{1}$

${ }^{1}$ Department of Neurology, ${ }^{2}$ Department of Neuroradiology, ${ }^{3}$ Institute for Clinical Chemistry,

Universitätsmedizin Mannheim, University of Heidelberg, Mannheim, Germany

\begin{abstract}
Background: Statins have been proven to reduce the risk of stroke in primary and secondary prevention. These lipid-lowering agents reduce cholesterol deposition in atherosclerotic plaques, which would lead to slower growth of plaques. Beyond these direct effects on total and LDL-cholesterol, effects on other pathways have been implied to be involved in the protective effects of statins. on total and LDL-cholesterol, effects on other pathways have been implied to be involved in the protective effects of statins.
Proposed mechanisms include reduced clot formation and clot adherence to ruptured atherosclerotic plaques. We therefore Proposed mechanisms include reduced clot formation and clot adherence to ruptured atherosclerotic plaques. We therefore
hypothesized that statin pre-treatment would lead to different lesion patterns on DWI-MRI and clinical courses in acute ischemic stroke of atherosclerotic origin.

Methods: We identified all patients with acute, anterior circulation ischemic stroke and TIA with ipsilateral carotid artery stenosis treated in our centre in a 3-year-period. Patient charts were reviewed for relevant data, especially clinical course, comorbidity, medication, and degree of stenosis. The latter was determined by ultrasound and graded according to the criteria proposed by the German Society for Ultrasound in Medicine (DEGUM). When records were incomplete, patients were excluded. Two experienced raters (AF \& KS) evaluated all patient to determine the number and pattern of DWI lesions. Lesion numbers were categorized as $<5$, 5-10, and $>10$. Patterns were categorized as either embolic, hemodynamic, DWI-negative, or "other" (see Figure 1 for example of embolic and hemodynamic lesion patterns). Fisher's exact test was used to test for significant differences in categorical data, Wilcoxon's rank-sum test for numerical data.
\end{abstract}

Results: 224 patients were included, 137 of them with higher grade stenoses ( $\geq 70 \%$ ). Table 1 summarises patient characteristics. In comparison to patients not treated with statins, patients taking lipid-lowering medication exhibited a significantly higher incidence of hypertension $(p<0.001)$, diabetes $(p<0.05)$, hyperlipidemia $(p<0.05)$, previous stroke $(p<0.01)$, heart disease $(p<0.001)$, or peripheral artery disease $(p<0.01)$. On the other hand, they were also more likely to receive aspirin treatment prior to the current event $(p<0.001)$. The level of both total serum cholesterol and LDL was significantly lower in the statin-treated group $(p<0.001)$.

As shown previously, the incidence of a certain stroke pattern was clearly dependent on the degree of stenosis (Figure 2, $\mathrm{p}=0.008$ ). However, there was also a statistical interaction between statin treatment and stroke pattern, manifested in more DWI-negative and fewer hemodynamic infarctions in the statin-treated group (Figure $3, p<0.005$ ), as well as between statin treatment and the number of DWI lesions, expressed in fewer lesions in the statin group $(p<0.05)$. While this may in part be mediated by concomitant treatment with anti-platelet agents, there was also significant correlation in the subset of patients who received statins but not anti-platele agents (Figure 4, $p=0.03$ ). However, numbers were low in this group. The mean lesion diameter did not differ between statin treated and untreated patients.

Discussion: As was to be expected, patients who received statins were generally older and had more cardiovascular risk factors, while their cholesterol levels were lower. Many of them were also on an anti-platelet agent, which makes it difficult to interpret the results. With this caveat in mind, our data suggest that statins will influence stroke patterns with smaller numbers of lesions and less hemodynamic and more DWI-negative lesions. While higher proportions of TIAs and DWI-negative strokes are consistent with the proposed antithrombotic features of statins, these features also predict fewer embolic, but not necessarily fewer hemodynamic strokes. This may suggest that other statin effects play a role. E $g$, statins have been shown to improve cerebral vasomotor reactivity in animal models which would protect against hemodynamic infarcts. The lower number of lesions may well be a consequence of fewer hemodynamic strokes which will commonly consist of many small lesions.

We were not able to demonstrate a better clinical outcome for patients pre-treated with statins. This Is in contrast to other groups and may well be to a relatively small sample size and large heterogeneity within groups.

Conclusion: We present evidence of the effects of statin pre-treatment on lesion patterns in acute ischemic stroke of atherosclerotic origin. Further research should proceed with greater patient numbers and try to separate effects of statin pre-treatment and other protective agents, such as anti-platelet and anti-hypertensive agents.

\begin{tabular}{|c|c|c|c|c|c|c|}
\hline \multirow[t]{2}{*}{ Variable } & \multicolumn{3}{|c|}{ All stenosis grades } & \multicolumn{3}{|c|}{ Stenosis grade $>=\mathbf{7 0} \%$} \\
\hline & $\begin{array}{l}\text { Statin } \\
\text { pretreatment }\end{array}$ & $\begin{array}{l}\text { No statin } \\
\text { pretreatment }\end{array}$ & $p$ value & $\begin{array}{l}\text { statin } \\
\text { pretreatment }\end{array}$ & $\begin{array}{l}\text { No statin } \\
\text { pretreatment }\end{array}$ & $p$ value \\
\hline No. of patients & 74 & 150 & & 42 & 95 & \\
\hline Age, $y$, mean $( \pm S D)$ & $72.5(8.4)$ & $69.2(11.0)$ & 0.05 & $71.6(8.3)$ & $67.7(11.3)$ & $<0.05$ \\
\hline Men, \% & 74 & 63 & 0.01 & 76 & 66 & 0.31 \\
\hline Hypertension, \% & 93 & 75 & $<0.001$ & 98 & 72 & $<0.001$ \\
\hline Diabetes, $\%$ & 39 & 27 & 0.09 & 31 & 25 & 0.53 \\
\hline Hyperlipidemia, \% & 68 & 45 & $<0.01$ & 67 & 44 & $<0.01$ \\
\hline Current Smoking, \% & 59 & 61 & 0.89 & 45 & 45 & 1 \\
\hline Alcohol consumption, \% & 7 & 13 & 0.18 & 7 & 18 & 0.12 \\
\hline Previous TIA, \% & 1 & 2 & 1 & 0 & 0 & \\
\hline Previous stroke, \% & 26 & 11 & $<0.01$ & 21 & 8 & $<0.05$ \\
\hline Coronary heart disease, $\%$ & 49 & 10 & $<0.001$ & 50 & 9 & $<0.001$ \\
\hline Peripheral artery disease, $\%$ & 24 & 11 & $<0.01$ & 24 & 13 & 0.13 \\
\hline Pre-stroke aspirin & $88 \%$ & $29 \%$ & $<0.001$ & $88 \%$ & $28 \%$ & $<0.001$ \\
\hline Total serum cholesterol, $\mathrm{mg} / \mathrm{dl},( \pm S D)$ & $169.7(39.6)$ & $207.4(45.9)$ & $<0.001$ & $160.5(35.7)$ & $211.4(50.6)$ & $<0.001$ \\
\hline$L D L \mathrm{mg} / \mathrm{dl},( \pm S D)$ & \begin{tabular}{|l|}
$101.9(37.0)$ \\
\end{tabular} & $137.0(40.3)$ & $<0.001$ & $92.7(32.9)$ & $140.7(44.0)$ & $<0.001$ \\
\hline HDL mg/dl, ( $\pm S D)$ & $46.5(13.6)$ & $46.6(14.2)$ & 0.88 & $46.5(15.0)$ & $45.7(15.1)$ & 0.59 \\
\hline NIHSS at admission, median (range) & $2(0-23)$ & $4(0-28)$ & 0.14 & $4(0-23)$ & $4(0-21)$ & 0.67 \\
\hline NIHSS at discharge, median (range) & $1(0-23)$ & $2(0-26)$ & 0.50 & $3(0-23)$ & $2(0-26)$ & 0.67 \\
\hline Pre-stroke mRS, median (range) & $0(0-4)$ & $0(0-5)$ & 0.39 & $0(0-4)$ & $0(0-5)$ & 0.26 \\
\hline mRS at discharge, median (range) & $2(0-6)$ & $2(0-6)$ & 0.40 & $2.5(0-6)$ & $3(0-6)$ & 0.77 \\
\hline Mean lesion diameter, $m m$, mean, $( \pm S D)$ & $20.3(21.2)$ & $22.2(20.8)$ & 0.26 & $25.8(22.8)$ & $22.8(18.0)$ & 0.63 \\
\hline No. of DWI lesions, $\%$ & & & $<0.05$ & & & $<0.05$ \\
\hline$<5$ & 55.2 & 36.3 & & 50 & 25.2 & \\
\hline $5-10$ & 19.0 & 22.2 & & 28.1 & 28.7 & \\
\hline$>10$ & 25.9 & 41.4 & & 21.9 & 46.0 & \\
\hline Stroke pattern & & & $<0.05$ & & & $<0.05$ \\
\hline Embolic & 50.0 & 54.0 & & 58.5 & 57.9 & \\
\hline Hemodynamic & 18.1 & 29.3 & & 16.7 & 32.6 & \\
\hline DWI-negative & 26.4 & 11.3 & & 19.0 & 5.3 & \\
\hline Other & 5.5 & 6.0 & & 4.8 & 4.2 & \\
\hline
\end{tabular}

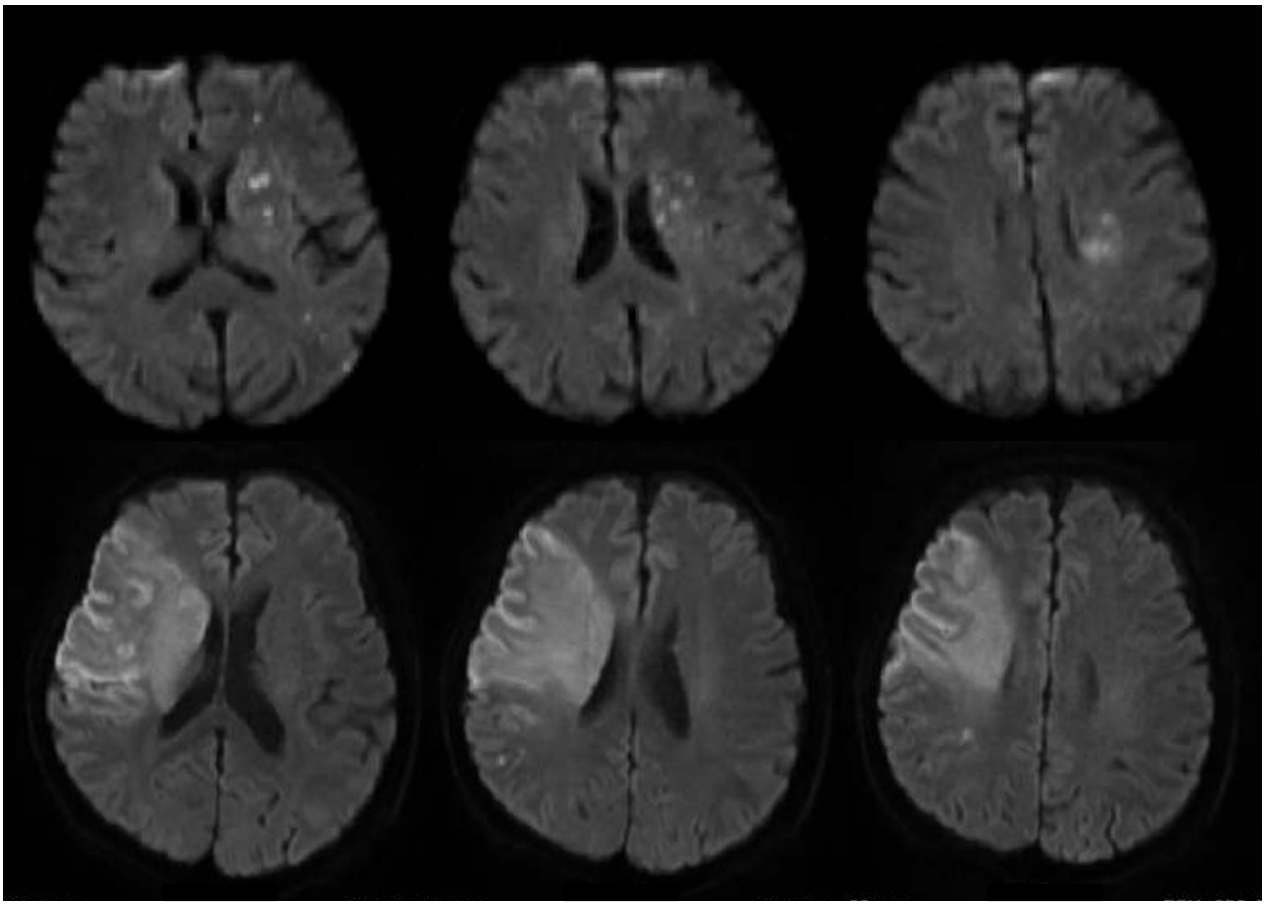

Figure 1: Examples of hemodynamic (upper row) and embolic (lower row) patterns of stroke

Degree of stenosis and stroke patterns

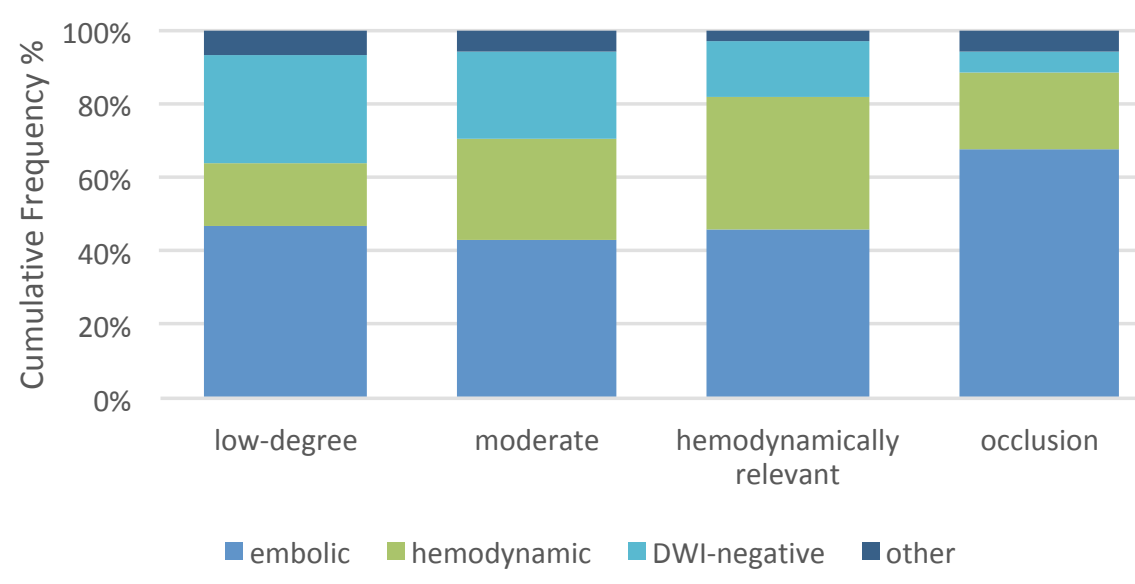

Figure 2: Interaction between degree of stenosis and lesion pattern

Statin pre-treatment and stroke patterns (士anti-platelets)

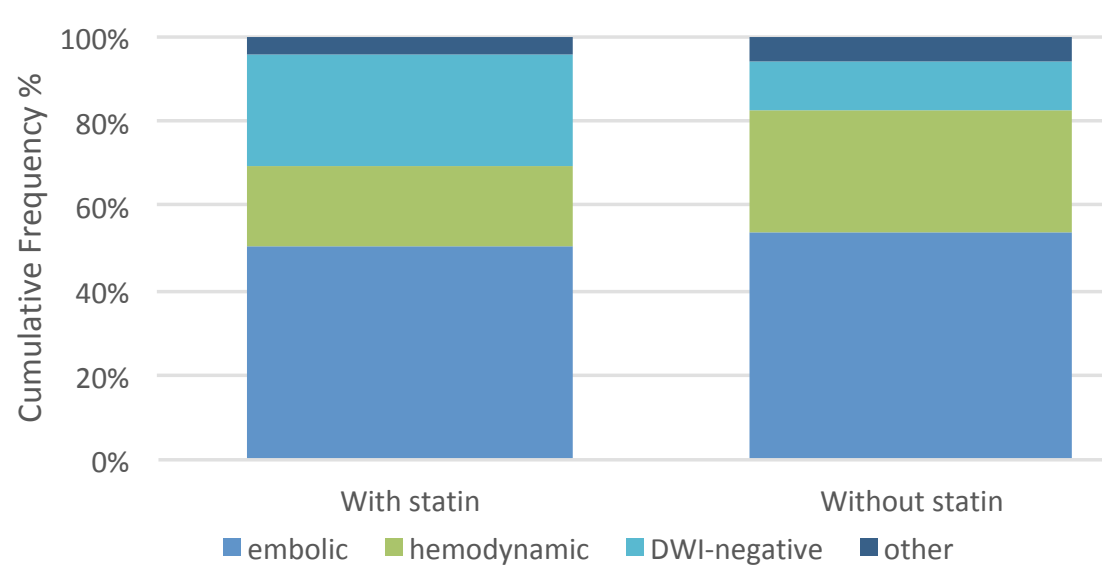

Figure 3: Patients treated with statins have fewer hemodynamic strokes

Statin pre-treatment and stroke patterns (without anti-platelets)

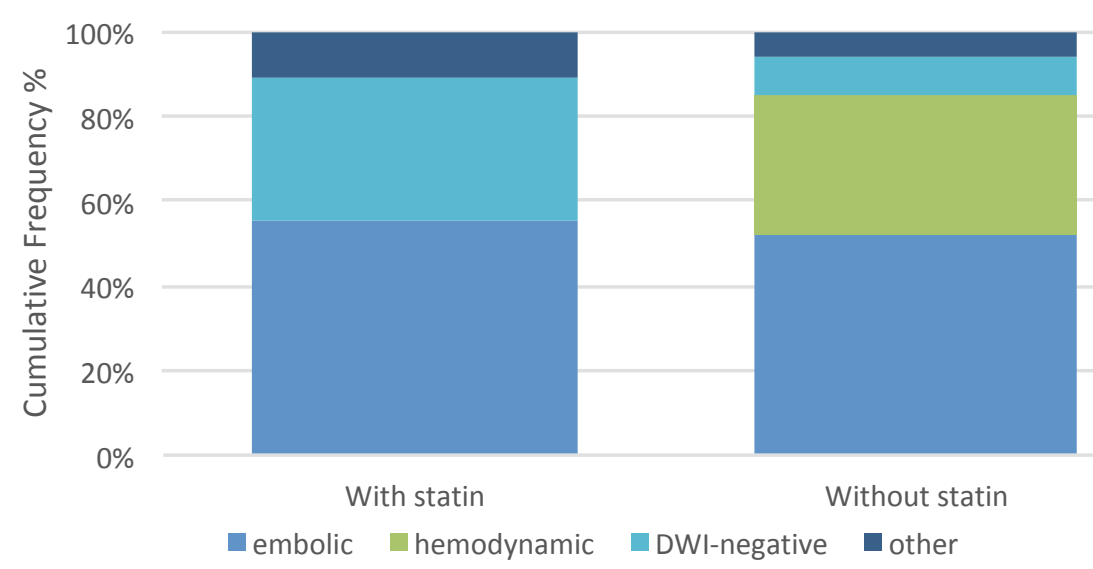

Figure 4: The effect of statins on stroke patterns is apparent also in patients with no prior anti-platelet treatment 